\title{
LIF is more potent than BDNF in promoting neurite outgrowth of mammalian auditory neurons in vitro
}

\author{
Lisa N. Gillespie, ${ }^{\text {CA }}$ Graeme M. Clark, Perry F. Bartlett' ${ }^{\prime}$ and Phillip L. Marzella \\ Department of Otolaryngology, The University of Melbourne, Royal Victorian Eye and Ear Hospital, 32 Gisborne Street, East \\ Melbourne, Australia 3002; 'Development and Neurobiology Laboratory, The Walter and Eliza Hall Institute of Medical \\ Research, Parkville, Australia 3052

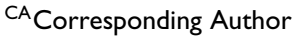

Received 27 October 2000; accepted 16 November 2000

Neurotrophic factors are known to play a crucial role in the elongation and guidance of auditory nerve fibres to their targets within the organ of Corti. Maintenance of these neural connections following deafness would clearly influence the efficacy of therapies for hearing recovery. The growth factors leukaemia inhibitory factor (LIF), brain-derived neurotrophic factor (BDNF) and transforming growth factor-beta 5 (TGF$\beta 5)$ were tested for their efficacy in promoting neurite outgrowth from dissociated cultures of early postnatal rat

Key words: Auditory; BDNF; LIF; Mammalian; Neurite outgrowth auditory neurons. Our results indicate that while BDNF enhances neurite outgrowth in a strong fashion, LIF is more potent; moreover, the combined administration of both factors has even greater neuritogenic capacities. TGF- $\beta 5$, although neurotrophic, has no neuritogenic activity on cultured auditory neurons. LIF and BDNF may therefore be potential candidates when developing pharmacological therapies for hearing recovery. NeuroReport 12:275-279 (C) 200I Lippincott Williams \& Wilkins.

\section{INTRODUCTION}

In the auditory system, primary innervation of the auditory hair cells involves the growth of neurites from the spiral ganglion towards the developing cochlear duct, followed by the establishment of precise connections between the hair cells and spiral ganglion neurons [1]. Neurotrophic factors, in particular nerve growth factors, produced and secreted by the hair cells, are known to play a crucial role in the elongation and guidance of auditory nerve fibres to their appropriate target within the organ of Corti [1,2]. In particular, the neurotrophins BDNF and neurotrophin-3 (NT-3) have been identified as essential for the normal development and innervation of the inner ear [1,3]. Indeed, studies using in situ hybridization have revealed higher expression of neurotrophins on hair cells during the period when spiral ganglion cells (SGCs) send their processes to these epithelia, and at the same time spiral ganglion neurons have been found to express mRNA for the high-affinity neurotrophin receptors trkB and trkC [4]. Further developmental studies of double mutant mice have reported that absence of both BDNF and NT-3 or of trkB and trkC leads to a nearly complete loss of inner ear neurons [3] and a complete loss of all afferent innervation of the ear [5]. These studies provide strong evidence that the neurotrophins are retrogradely transported from hair cells to the SGCs to influence development and survival, and importantly, regulate sy- naptic connections between hair cells and SGCs for the onset of auditory function.

Neurotrophic factors have also been demonstrated to play a role in neuritogenesis and synaptic connectivity. The delivery of either BDNF or NT-3 can stimulate neurite outgrowth from early postnatal auditory neurons in dissociated cultures [6], and also promote neuritogenesis in explant cultures of embryonic cochlear ganglia [2,7], suggesting a role for neurotrophins in modulating neuritic outgrowth from SGCs. Other trophic factors have also been demonstrated to induce neurite outgrowth from auditory neurons, including the cytokine ciliary-derived neurotrophic factor (CNTF). CNTF has been reported to have neuritogenic effects on organotypic explant cultures of early postnatal auditory neurons, and can also act in an additive fashion with BDNF to further enhance neurite outgrowth [7].

Previously, we have reported that the neuronal cytokine leukaemia inhibitory factor (LIF), which is structurally homologous to $\mathrm{CNTF}$, is a survival factor for auditory neurons in vitro; moreover, LIF acts synergistically with BDNF to further support the survival of cultured SGCs [8]. We have also observed that members of the transforming growth factor-beta (TGF- $\beta$ ) family of growth factors support the survival of cultured SGCs [9], and in particular TGF- $\beta 5$ acts in a synergistic manner with BDNF [8].

In the present study, we have quantitatively assessed the effects of several growth factors, both individually and in 
combination, for their efficacy in promoting neurite outgrowth from early postnatal rat auditory neurons grown in vitro.

\section{MATERIALS AND METHODS}

Cultures of mammalian auditory neurons were prepared from 5-day-old Sprague-Dawley rat pups. The rat pups were anaesthetized on ice until unconscious, rapidly decapitated and the heads rinsed in $70 \%$ ethanol. Under aseptic conditions the skull was opened longitudinally, the temporal bone identified and the bulla removed. In a solution of HEPES buffered Eagle's media (HEM) the cochleae was isolated, and the organ of Corti carefully removed. The modiolus of the cochleae was then digested in HEM containing $0.025 \%$ trypsin (Calbiochem) and $0.001 \%$ DNase (Boehringer Mannheim) at $37^{\circ} \mathrm{C}$ for 30 minutes. Trypsinization was terminated by addition of $1 \mathrm{ml}$ fetal calf serum (FCS), and the digested cochleae centrifuged at $1500 \times g$ for $10 \mathrm{~min}$ at room temperature. The supernatant was discarded and the tissue was then mechanically digested by trituration in a solution of HEM containing $0.001 \%$ DNase. The cell suspension was again spun at $1500 \times g$ for $10 \mathrm{~min}$ and the resultant pellet was resuspended in Dulbecco's modified Eagle's medium (DMEM) with N1 supplement and $4.5 \mathrm{~g} / 1$ glucose. The dissociated SGC suspension was pre-plated on a $35 \mathrm{~mm}$ tissue culture plate and incubated for $30 \mathrm{~min}$ at $37^{\circ} \mathrm{C}$ with $10 \% \mathrm{CO}_{2}$. The neuron-enriched cell suspension was collected and a cell count performed. The cells were plated at a density of 10000 cells/well onto a 96-well tissue culture plate coated with poly-ornithine $(500 \mu \mathrm{g} / \mathrm{ml})$ and laminin $(0.01 \mathrm{mg} / \mathrm{ml})$. Growth factors were added at a final concentration of $50 \mathrm{ng} / \mathrm{ml}$, since previous studies have found that BDNF, LIF and TGF- $\beta 5$ have maximal survival-promoting effects when used at this concentration [8]. Cultures were maintained for 3 days at $37^{\circ} \mathrm{C}$ with $10 \% \mathrm{CO}_{2}$. In order to determine the initial number of neurons plated, a separate tissue culture dish was prepared and cultured for $4 \mathrm{~h}$ without growth factors, termed the seed plate. At the end of the appropriate culture periods the cells were fixed in $100 \%$ ice-cold methanol and immunostained.

Immunostaining was performed using the avidin-biotin complex (ABC) method of a standard VectaStain kit (Vector). Following fixation, the cultures were rinsed with phosphate buffered saline (PBS), and then pretreated with $2 \%$ FCS in PBS for $30 \mathrm{~min}$ at room temperature. The cultures were then incubated with rabbit anti-neurofilament $200 \mathrm{kDa}$ at 1:400 dilution for $30 \mathrm{~min}$ at room temperature, rinsed with $2 \%$ FCS solution and treated with the biotinylated anti-rabbit antibody (1:200 dilution) for $30 \mathrm{~min}$ at room temperature. The cells were then washed in PBS and reacted with $\mathrm{ABC}$ as per the manufacturers' instructions. Finally, cultures were reacted with diaminobenzidene (DAB; DAKO), prepared as 1 tablet in $10 \mathrm{ml} \mathrm{PBS}$ with $30 \mu \mathrm{l}$ hydrogen peroxide.

Surviving neurons were defined as those whose somas stained against the neurofilament antibody, and with neuritic projections that were at least equal in length to three times the width of the neuronal soma. Measurements were made of neuronal length using the software package Scion image (NIH). The length of the primary axon was measured from the edge of the soma to its termination; the primary axon was identifiable as the thicker and generally more heavily stained projection. Soma size was also measured, and the number of branching points was also counted.

Neurons were measured from five fields of view within each well, with one neuron selected within each field (ie. 5 neurons/well). For those fields of view with more than one neuron, the neuron for measurement was selected based upon ability to follow the primary axon to its termination, as many neurons had quite convoluted projections that were difficult to trace. For those wells with less than 5 neurons in total, all neurons were measured, despite their location within the well. It should also be noted that it was the cell bodies of the neurons that were located within the respective field of view, regardless of where the neurites extended. The fields of view were consistent between wells, and were such that four were located around the perimeter of the well, one each in the centre of each side and top and bottom, with the remaining field of view in the centre of the well (Fig. 1).

Data were analysed statistically using ANOVA, and Dunn's method was used for an all pairwise multiple comparison procedure. A difference was considered to be statistically significant at $p<0.05$.

The care and use of animals reported on in this study was approved by the Animal Research Ethics Committee of the Royal Victorian Eye and Ear Hospital, and experiments were performed in accordance with the NH\&MRC of Australia.

\section{RESULTS}

SGCs were plated at a density of $1 \times 10^{4}$ cells/well, with a mean ( \pm s.e.m.) seed number of $497 \pm 26$ neurons/well. SGCs grown in culture without the addition of any growth factors (control wells) had primary axons that measured $588 \pm 43 \mu \mathrm{m}$. The addition of LIF to the auditory neuron cell cultures significantly enhanced the elongation of the primary axons $(p<0.05)$, with an average length of $1435 \pm 85 \mu \mathrm{m}$, a 2.4 -fold increase compared with the average length of primary axons of neurons in the control wells. Treatment with BDNF also had a significant effect

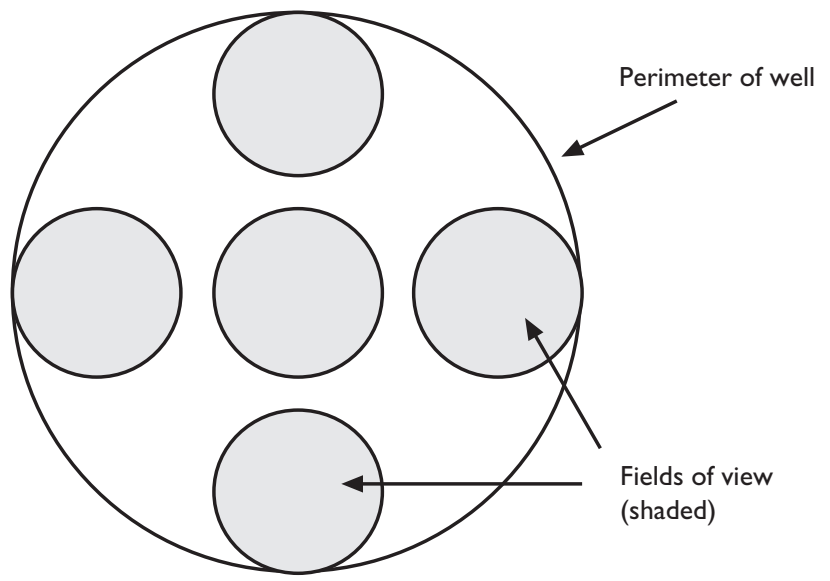

Fig. I. Schematic representation of the fields of view from which neurons were selected for measurement. The primary axon of one neuron within each field of view was measured. 
on the length of primary projections from SGCs $(p<0.05)$, increasing the length of these projections to $925 \pm 32 \mu \mathrm{m}$, an $\sim 1$.6-fold increase when compared to control. The addition of TGF- 35 , although promoting survival, did not significantly enhance elongation of the primary axons of SGCs in culture compared with control wells $(p<0.05)$, with an average length of neuronal projections of $654 \pm 20 \mu \mathrm{m}$ (Fig. 2).

When we co-administered LIF and BDNF extensive neuritic outgrowth was observed (Fig. 3). In these wells we could not discern or follow the individual projections of any one neuron, nor could we measure the length of these neuronal projections. This was true for each experiment in which LIF plus BDNF was a treatment factor: a total of seven experiments, with four wells per experiment and an average of $375 \pm 30$ neurons/well. We also co-administered BDNF and TGF- 35 ; neuritic outgrowth was not significantly different from that observed with BDNF alone $(p<0.05)$. The average length of the primary neuronal projection following concurrent treatment with BDNF and TGF- $\beta 5$ was $883 \pm 22 \mu \mathrm{m}$, a 1.5 -fold increase compared with controls.

Analysis of the effects of trophic factor treatment was also made for soma size and the number of branching points. None of the growth factors had a significant effect on either parameter (data not shown).

\section{DISCUSSION}

We report that both LIF and BDNF promote neurite outgrowth from early postnatal auditory neurons cultured in vitro. BDNF induces a 1.6-fold increase in the average length of the primary axon when compared to control wells. In addition, we observed that LIF had a stronger neuritogenic effect than BDNF, resulting in a 2.4-fold increase in average primary axon length as compared to controls. Co-administration of these two factors led to such

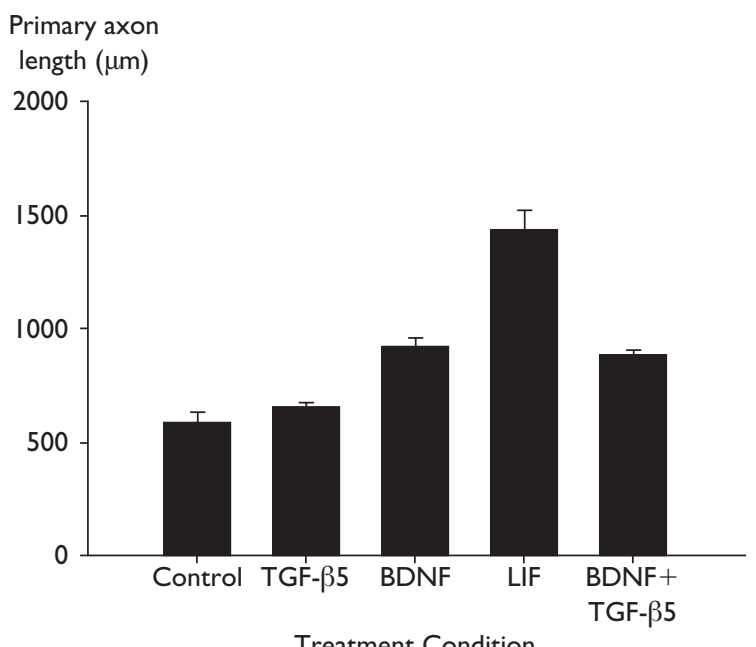

Fig. 2. Effects of growth factors on the length of primary axons of P5 rat auditory neurons after $72 \mathrm{~h}$ in vitro. The final concentration of each growth factor is $50 \mathrm{ng} / \mathrm{ml}$. The results are expressed as average primary axon length $(\mu \mathrm{m})$ for the various treatment conditions, with mean \pm s.e.m. A total of 100 neurons per treatment were measured over five experiments, with four wells per experiment and five neurons per well. extensive neuritic outgrowth that it was impossible to trace individual axons of any one neuron for analysis. In contrast, TGF- $\beta 5$ did not significantly enhance neuritic outgrowth beyond that measured in control wells, and combined delivery of TGF- $\beta 5$ with BDNF was no more effective than BDNF alone.

Several trophic factors have previously been reported to be involved in both neuritogenesis and synaptogenesis of developing and maturing neurons throughout the nervous system; however, the exact mechanisms by which they regulate or stimulate neuritogenesis remain unknown. Several possible lines of evidence may provide explanation for our current results.

First, axonal elongation depends upon the dynamics and structure of the actin cytoskeleton of the neuronal growth cone [10]. Many mechanisms have been either identified or speculated as controlling factors of the neuronal cytoskeleton. One factor known to regulate actin assembly is RhoA, a member of the Ras superfamily of GTP-binding proteins [11], which has been identified to mediate growth cone collapse [12]. Recent studies have found that the common neurotrophin receptor, p75, interacts in a ligand-dependent manner with RhoA, and inactivation of Rho proteins has been reported to increase the rate of neurite elongation [13]. Therefore, BDNF signalling through the p75 receptor may alter the organization and dynamics of actin assembly and affect neurite outgrowth by regulating the activity of Rho.

Secondly, both LIF and BDNF may regulate neuritogenesis through altering calcium levels. Evidence suggests that intracellular calcium transients can govern growth cone motility and axonal growth [14]. Both LIF and the neurotrophins regulate protein kinase C (PKC) activity [15,16], and activation of PKC is known to mediate intracellular calcium concentrations [17]. Furthermore, BDNF has been suggested to have a close relationship with calcium-binding proteins [18]. Taken together, these findings suggest that both LIF and BDNF may affect axonal outgrowth through regulation of intracellular calcium levels.

Finally, LIF and BDNF share similar intracellular effectors. Indeed, converging intracellular pathways acting downstream from LIF and BDNF have been suggested to underpin the synergy between these two growth factors when promoting survival [8]. Such convergence may also play a role in neuritic outgrowth. LIF and the neurotrophins may interact via the Ras-MAP-kinase (Ras-mitogen activated protein kinase) intracellular signalling pathway, through phosphatidyl-inositol-3 (PI-3) activation [19,20], or even through the STAT (signal transducers and activators of transcription) pathway, since both LIF and the neurotrophins have been indicated to regulate tyrosine phosphorylation and STAT activation $[19,21]$.

We also report that TGF- $\beta 5$, although neurotrophic, is not a neuritogenic factor for auditory neurons in culture. TGF- $\beta 5$ alone did not enhance neuritic outgrowth significantly above that of controls, and co-administration with BDNF was no more effective than BDNF alone. Interestingly, the TGF- $\beta$ s are known to regulate similar signalling pathways to LIF, namely, PKC and PI-3 turnover [22], as well as eliciting some intracellular signalling via the Smad proteins, which in turn are reported to interact with the Ras-MAP-kinase pathway [23]. The observation that exo- 

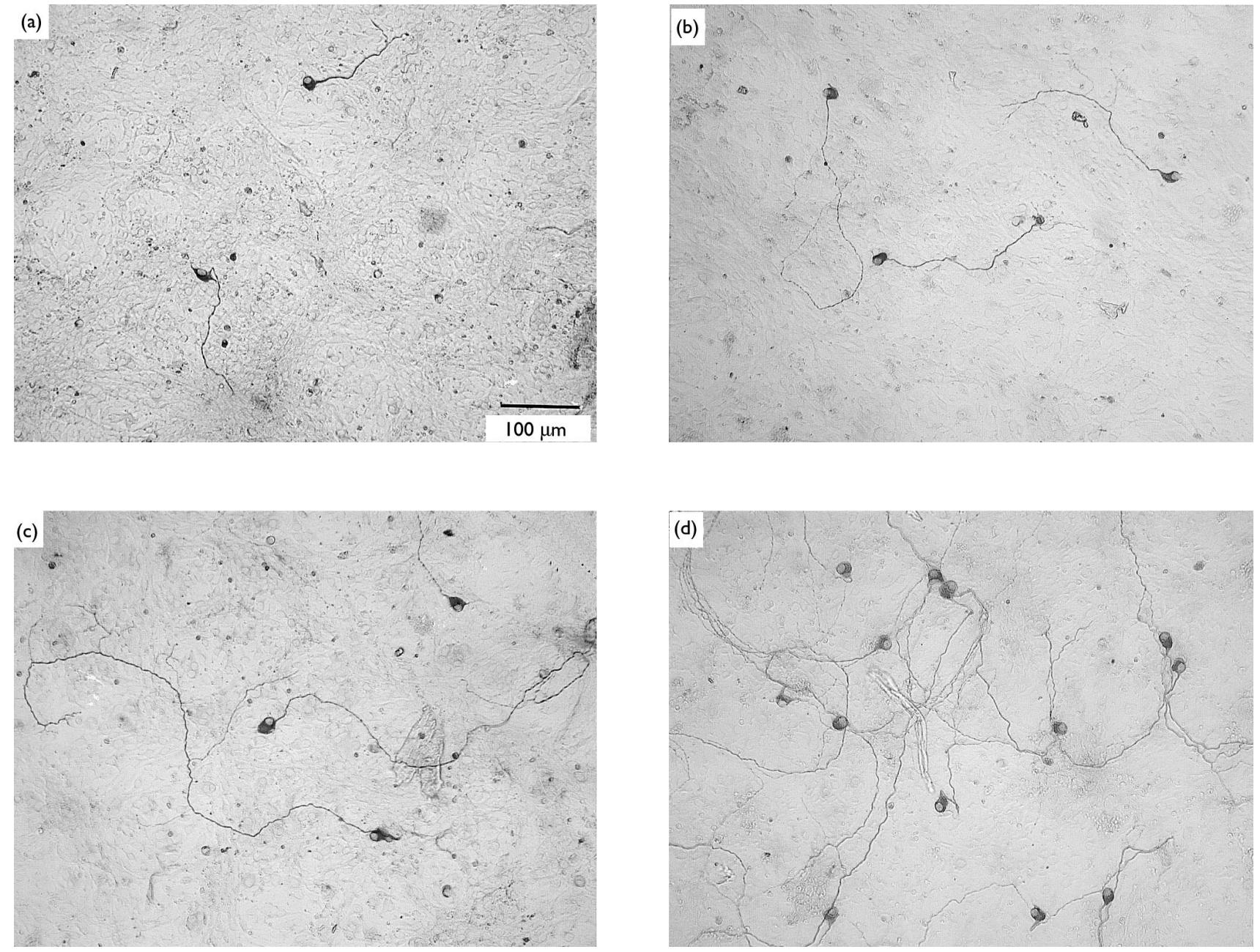

Fig. 3. Neuritogenic effects of several growth factors on cultured P5 rat auditory neurons. (a) Control, no growth factors; (b) BDNF; (c) LIF; (d) $\mathrm{LIF}+\mathrm{BDNF}$. The final concentration of each growth factor is $50 \mathrm{ng} / \mathrm{ml}$.

genous LIF can elicit strong neuritic outgrowth from auditory neurons, while TGF- $\beta 5$ does not have this capacity, suggests that perhaps the activation of the STAT signalling pathway by LIF is an important signalling pathway for neuritogenesis. In addition, LIF is also a mediator of Schwann cell survival [24], and so may exert indirect survival effects on SGCs. These results suggest that different but overlapping mechanisms may regulate survival and neuritogenesis.

\section{CONCLUSION}

We have identified LIF as a potent neuritogenic factor for auditory neurons grown in culture, and have supported previous studies identifying a similar role for BDNF. BDNF enhances neurite outgrowth in a strong fashion, however LIF is more potent, and co-administration of these two factors resulted in extensive neuritic outgrowth, past the point of analysis. In the auditory system, the maintenance of neural connections from the auditory neurons to the brain following deafness may allow for the development of pharmacological therapies for deafness. LIF and BDNF may therefore be potential candidates for such pharmacological therapy.

\section{REFERENCES}

1. Despres G and Romand R. Life Sci 54, 1291-1297 (1994).

2. Avila MA, Varela-Nieto I, Romero G et al. Dev Biol 159, 266-275 (1993).

3. Ernfors P, Van De Water T, Loring J et al. Neuron 14, 1153-1164 (1995).

4. Pirvola U, Arumae U, Moshnyakov M et al. Hear Res 75, 131-144 (1994).

5. Fritzsch B, Silos-Santiago I, Bianchi LM et al. Semin Cell Dev Biol 8, 277-284 (1997).

6. Malgrange B, Lefebvre PP, Martin D et al. Neuroreport 7, 2495-2499 (1996).

7. Hartnick CJ, Staecker H, Malgrange B et al. J Neurobiol 30, 246-254 (1996).

8. Marzella PL, Gillespie LN, Clark GM et al. Hear Res 138, 73-80 (1999).

9. Marzella PL, Clark GM, Shepherd RK et al. Neurosci Lett 240, 77-80 (1998).

10. Gallo G and Letourneau PC. Curr Biol 9, R490-492 (1999).

11. Mackay DJ and Hall A. J Biol Chem 273, 20685-20688 (1998).

12. Caroni P. Science 281, 1465-1466 (1998).

13. Yamashita T, Tucker KL and Barde YA. Neuron 24, 585-593 (1999).

14. Gomez TM and Spitzer NC. I Neurobiol 44, 174-183 (2000).

15. Maness LM, Kastin AJ, Weber JT et al. Neurosci Biobehav Rev 18, 143-159 (1994).

16. Matsuoka I, Nakane A and Kurihara K. Brain Res 776, 170-180 (1997). 
17. Jiang H and Guroff G. J Neurosci Res 50, 355-360 (1997).

18. Iwasaki K, Isaacs KR and Jacobowitz DM. Int J Dev Neurosci 16, 135-145 (1998).

19. Gadient RA and Patterson PH. Stem Cells 17, 127-137 (1999).

20. Ip NY and Yancopoulos GD. Annu Rev Neurosci 19, 491-515 (1996).
21. Rajan P, Gearan T and Fink JS. Brain Res 802, 198-204 (1998).

22. Segarini PR. Ciba Found Symp 157, 29-40 (1991).

23. Kretzschmar M and Massague J. Curr Opin Genet Dev 8, 103-111 (1998).

24. Dowsing BJ, Morrison WA, Nicola NA et al. J Neurochem 73, 96-104 (1999).

Acknowledgements: L.N.G. is a recipient of a Research Scholarship from The Eye, Ear, Nose and Throat Research Institute,

Melbourne, Australia. P.L.M. is a recipient of a Wagstaff Research Fellowship in Otolaryngology from the Department of

Otolaryngology, The University of Melbourne. G.M.C. is funded by the Human Communication Research Centre of the

Department of Otolaryngology, The University of Melbourne. P.F.B. is funded by the NH and MRC of Australia. 


\section{University Library}

\section{- M M N E R VA A gateway to Melbourne's research publications}

Minerva Access is the Institutional Repository of The University of Melbourne

Author/s:

Gillespie, Lisa N.;Clark, Graeme M.;Bartlett, Perry F.;Marzella, Phillip L.

Title:

LIF is more potent than BDNF in promoting neurite outgrowth of mammalian auditory neurons in vitro

Date:

2001

Citation:

Gillespie, L. N., Clark, G. M., Bartlett, P. F., \& Marzella, P. L. (2001). LIF is more potent than BDNF in promoting neurite outgrowth of mammalian auditory neurons in vitro. NeuroReport, February, 12(2), 275-279.

Persistent Link:

http://hdl.handle.net/11343/27588 